\title{
An increase in the extracellular potassium concentration can cause seizures
}

\author{
Tianlin Ying ${ }^{1}$, David B Grayden ${ }^{1,2}$, Anthony N Burkitt ${ }^{1,2}$, Tatiana Kameneva ${ }^{\text {* }}$ \\ From 24th Annual Computational Neuroscience Meeting: CNS*2015 \\ Prague, Czech Republic. 18-23 July 2015
}

\begin{abstract}
Introduction
Epilepsy is a neurological disorder characterized by recurrent seizures. It is important for the development of patient treatments to understand the mechanisms underlying this complex neurological disease. Experimental data shows that an increase in the extracellular potassium concentration, $\left[\mathrm{K}^{+}\right]_{0}$, can support the generation of seizures and growth in seizures frequency and propagation velocity [1]. It is unclear if seizures are caused by the increase in $\left[\mathrm{K}^{+}\right]_{0}$ or seizures cause the $\left[\mathrm{K}^{+}\right]_{0}$ increase.
\end{abstract}

\section{Methods}

Using a single column model neural mass model of pyramidal cell population [2], we explore how the change in $\left[\mathrm{K}^{+}\right]_{0}$ affects the model output. The model represents the activity of approximately $10^{5}$ neurons and has four interacting neural populations: pyramidal neurons, excitatory, slowand fast-inhibitory neurons. The model output is interpreted as representing the recorded EEG signal. A static nonlinear function (a sigmoid) of the model converts a postsynaptic potential into an average spiking rate for each neural population. We fit data from [3] to different forms of the sigmoid corresponding to different concentration of $\left[\mathrm{K}^{+}\right]_{0}$. Simulations were run with different sigmoid parameters while other parameters of the model are fixed to produce normal background activity (non-seizure dynamics). To classify the model output as a particular neural activity type, a power spectral density analysis is used and the types were compared to those in [2].

\section{Results}

Results show that a $\left[\mathrm{K}^{+}\right]_{0}$ increase from 5 to $13 \mathrm{mM}$ causes the neural dynamics to transit from Type 1 (normal

\footnotetext{
* Correspondence: tkam@unimelb.edu.au

${ }^{1}$ NeuroEngineering Laboratory, Department of Electrical and Electronic

Engineering, University of Melbourne, Melbourne, Victoria 3010, Australia
}

Full list of author information is available at the end of the article background activity) to Type 6 (slow quasi-sinusoidal activity) to Type 3 (sustained discharge of spikes) to Type 1, and then to Type 5 (low-voltage rapid activity).

\section{Conclusions}

Our results confirm that a $\left[\mathrm{K}^{+}\right]_{0}$ increase can generate seizures. This may have implications on the development of the effective treatments for epilepsy patients. It is left for future research to investigate whether changes in $\left[\mathrm{K}^{+}\right]_{0}$ can affect the frequency or propagation velocity of seizures. These investigations will be carried out using multiple interconnected columns.

\section{Acknowledgements \\ This research was supported by the Australian Research Council (DE120102210). The Bionics Institute acknowledges the support it receives from the Victorian Government through its Operational Infrastructure Support Program.}

\section{Authors' details}

${ }^{1}$ NeuroEngineering Laboratory, Department of Electrical and Electronic Engineering, University of Melbourne, Melbourne, Victoria 3010, Australia. ${ }^{2}$ Bionics Institute, Melbourne, Victoria 3002, Australia.

\section{Published: 18 December 2015}

\section{References}

1. Jensen MS, Yaari Y: Role of intrinsic burst firing, potassium accumulation, and electrical coupling in the elevated potassium model of hippocampal epilepsy. J Neurophys 1997, 77:1224-1233.

2. Wendling F, Bartolomei F, Bellanger JJ, Chauvel P: Epileptic fast activity can be explained by a model of impaired GABAergic dendritic inhibition. Europ J Neurosci 2002, 9:1499-1508.

3. Zandt BJ, Visser S, van Putte MJAN, ten Haken B: A neural mass model based on single cell dynamics to model pathophysiology. J Comput Neurosci 2014, 37:549-568.

\section{doi:10.1186/1471-2202-16-S1-P113}

Cite this article as: Ying et al:: An increase in the extracellular potassium concentration can cause seizures. BMC Neuroscience 2015 16(Suppl 1): P113. 